\title{
Structure determination of low-molecular weight targets at near-atomic resolution using single- particle cryo-electron tomography
}

HSUAN-FU LIU ${ }^{1}$, Ye Zhou ${ }^{2}$, Xiaochen Du$^{3}$, Jonathan Bouvette ${ }^{4}$, Mario Borgnia ${ }^{4}$ and Alberto Bartesaghi ${ }^{3}$

${ }^{1}$ Duke University, Durham, North Carolina, United States, ${ }^{2}$ Duke University, North Carolina, United States, ${ }^{3}$ Duke University, United States, ${ }^{4}$ National Institute of Environmental Health Sciences, United States

Cryo-electron tomography (CET) combined with sub-volume averaging (SVA) is a powerful imaging technique for determining macromolecular structures in situ. To resolve structures at high-resolution, large numbers of sub-volumes containing the protein of interest are aligned and averaged in three dimensions by SVA. Using this strategy, the structures of highly-ordered virus capsid proteins and large ribosomes have been resolved at near-atomic resolution [1], [2]. However, CET studies of lower molecular weight targets (< $300 \mathrm{kDa}$ ) have been limited to sub-nanometer resolutions, due to limitations in the accuracy of image alignment resulting from the lower image contrast generated by the smaller scattering masses. One way to improve image alignment accuracy is to more precisely recover the signal modulated by the contrast transfer function (CTF). Previous studies have shown that determining accurate per-particle CTF values -derived from the z-distances to the central mean defoci- can significantly improve resolution [3]. However, existing techniques to estimate the mean defoci only use patches close to the tilt axis, resulting in less accurate defocus values. In addition, the lack of strategies to properly downweight the contribution of lower-quality high-tilt projections can also limit resolution. Here, we present strategies to precisely determine per-particle CTF by taking advantage of the predetermined tilt-series alignment parameters. Using all the data in each projection, we can estimate an astigmatic tilted CTF-model with better precision. We also use a data-driven exposure weighting routine that allows better recovery of the high-resolution signal. Using this approach, we improved the resolution of two benchmark ribosomal datasets to $5.6 \AA$ and $4.8 \AA$, respectively (Figure 1). We also determined the structure of a low molecular weight protein $(\sim 300 \mathrm{kDa})$ at $3.6 \AA$ resolution [4], indicating that targets in this size range are within the reach of cryo-ET (Figure 2). The availability of strategies for near-atomic resolution structure determination of biomedically important targets using cryoET, represents another step towards closing the resolution gap between high-resolution strategies used to study molecular assemblies reconstituted in-vitro and techniques for in-situ structure determination. 

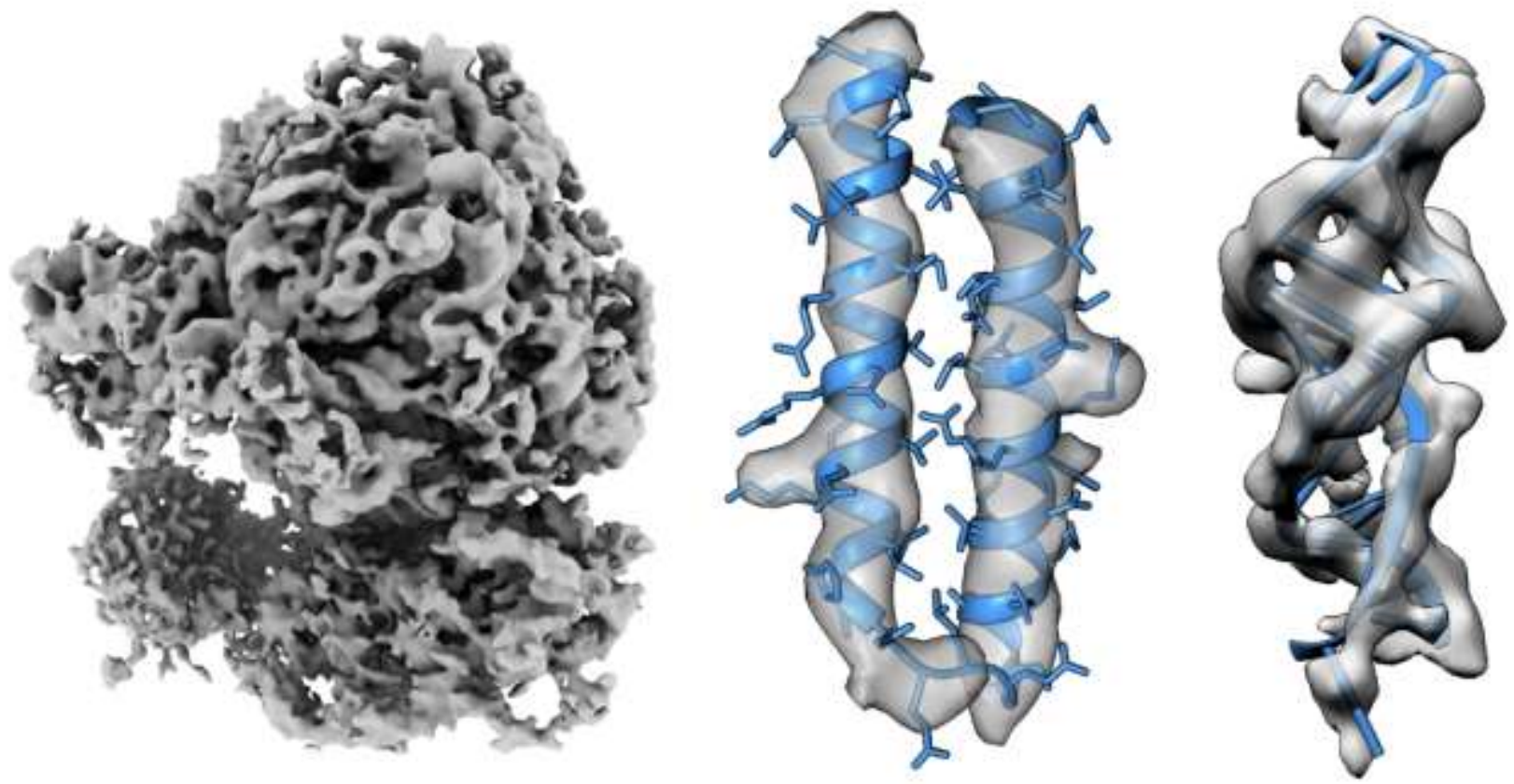

Figure 1. $4.8 \AA$ resolution structure from E. coli 70S Ribosome obtained from EMPIAR-10304. Overview of reconstruction (left), density for alpha-helical segments (middle), and for a segment of RNA (right), with atomic coordinates PDB ID 5MDZ fitted into the map. Original reported resolution for this dataset using emClarity was $7 \AA$. Resolutions were estimated using the 0.143 -FSC criteria.
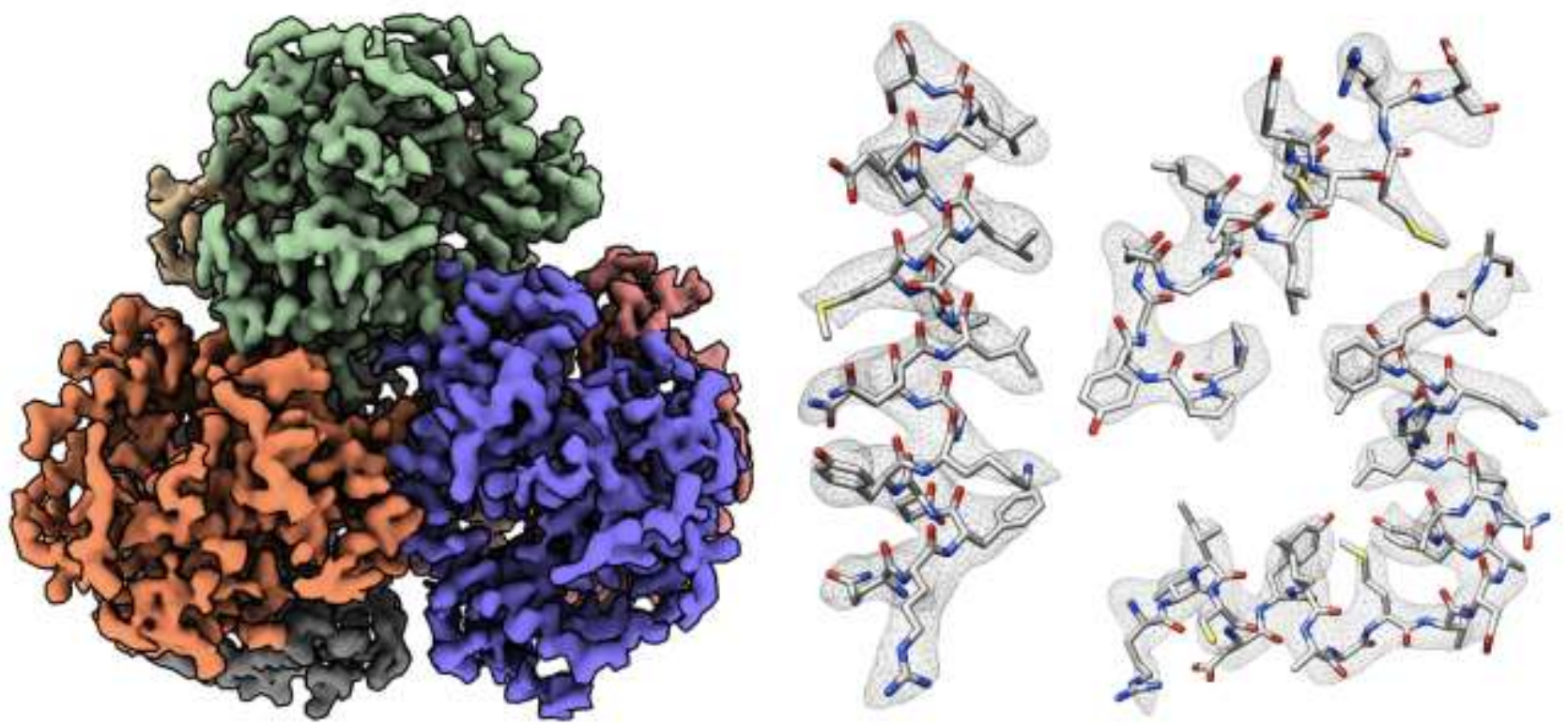

Figure 2. Near-atomic resolution cryo-ET structure of the low molecular weight target dNTPase. Overview of $3.6 \AA$ resolution map obtained from $\sim 30 \mathrm{k}$ particles extracted from 64 low-dose tomographic tilt-series 
(left). Total molecular weight is $\sim 300 \mathrm{kDa}$. Selected regions of the cryo-ET density map with fitted atomic coordinates show clear visualization of side chains (right).

\section{References}

[1]F. K. M. Schur et al., "An atomic model of HIV-1 capsid-SP1 reveals structures regulating assembly and maturation," Science, vol. 353, no. 6298, pp. 506-508, 2016.

[2]D. Tegunov, L. Xue, C. Dienemann, P. Cramer, and J. Mahamid, "Multi-particle cryo-EM refinement with M visualizes ribosome-antibiotic complex at $3.5 \AA$ in cells," Nature Methods, vol. 18, no. 2, Art. no. 2, Feb. 2021.

[3]A. Bartesaghi, F. Lecumberry, G. Sapiro, and S. Subramaniam, "Protein Secondary Structure Determination by Constrained Single-Particle Cryo-Electron Tomography," Structure, vol. 20, no. 12, pp. 2003-2013, Dec. 2012.

[4]J. Bouvetteet al., "Beam image-shift accelerated data acquisition for near-atomic resolution single-particle cryo-electron tomography," bioRxiv, p. 2020.09.24.294983, Jan. 2021. 